Pacific Journal of Mathematics

HE NORMAL INDEX OF A FINITE GROUP

MukHerjee and Prabir Bhattacharya 


\section{THE NORMAL INDEX OF A FINITE GROUP}

\section{N. P. Mukherjee AND Prabir Bhattacharya}

For a maximal subgroup $M$ of a finite group $G$ the normal index of $M$ is the order of a chief factor $H / K$ where $H$ is minimal in the set of supplements of $M$ in $G$. We obtain results about the normal index of $M$ when $M$ has composite index in $G$.

1. Introduction. The relationships between the properties of maximal subgroups of a finite group $G$ and the structure of $G$ have been studied by many people. In [3], [4] and [10] we investigated maximal subgroups of composite index, developing analogs of the Frattini subgroup and studying their role in the structure of groups. Here we obtain results which involve the normal index (introduced by Deskins in [5]) of a maximal subgroup $M$ of a group $G$. The normal index of $M, \eta(G: M)$, is the order of a chief factor $H / K$ of $G$ when $H$ is a minimal supplement of $M$ in $G$. In $\S \S 2-4$ we obtain extensions of results of Deskins [5], Beidleman and Spencer [2] and Mukherjee [9] based on $\eta(G: M)$ for the case when $[G: M]$ is composite.

All groups treated are finite, notation is standard (from [6] and [8]), and a maximal subgroup $M$ of $G$ is often denoted by $M<G$. If $M<\cdot G$ and $[G: M]$ is composite we call $M c$-maximal in $G$.

2. Normal index and solvability. If $M$ is a maximal subgroup of a group $G$ and $H$ is a minimal normal supplement to $M$ in $G$ then for any chief factor $H / K$ of $G$ it follows that $K \subseteq M$ and $G=M H$. Therefore we have that $[G: M]$ divides $o(H / K)=\eta(G: M)$. For the sake of completeness we first describe some properties of the normal index which we shall use subsequently.

2.1 (Deskins [5, 2.1], Beidleman and Spencer [2, Lemma 1]). If $M$ is a maximal subgroup of a group $G$ then $\eta(G: M)$ is uniquely determined by $M$.

2.2 (Beidleman and Spencer [2, Lemma 2]). If $N$ is a normal subgroup of a group $G$ and $M$ is a maximal subgroup of $G$ such that $N \subseteq M$ then $\eta(G / N: M / N)=\eta(G: M)$. 
It was announced by Deskins in $[5,2.5]$ that a group $G$ is solvable if and only if $\eta(G: M)=[G: M]$ for each maximal subgroup $M$ of $G$ (a detailed proof appears in [2]). We extend this theorem by proving the following:

Theorem 2.3. A group $G$ is solvable if and only if:

$$
\eta(G: M)=[G: M] \text { for every c-maximal subgroup } M \text {. }
$$

Proof. Let $\mathcal{J}$ be the family of all $c$-maximal subgroups of $G$. If $\mathscr{J}$ is empty then every maximal subgroup of $G$ has prime index and so $G$ is supersolvable (using a well known result of Huppert [7]) implying that $G$ is solvable, proving the result. So we may assume that $\mathscr{J}$ is non-empty. We use induction on the order of $G$. If $G$ is simple, then for any $M \in \mathcal{J}$ we have that $\eta(G: M)=o(G)=[G: M]$ implying that $o(M)=1$, a contradiction. Therefore $G$ is not simple. Now, suppose if possible, $N_{1}, N_{2}$ are two distinct minimal normal subgroups of $G$. Using 2.2 it is easy to see that the hypothesis holds for $G / N_{1}$ and $G / N_{2}$ and so by induction $G / N_{1}$ and $G / N_{2}$ are solvable implying that $G /\left(N_{1} \cap N_{2}\right)$ is solvable. Thus $G$ is solvable since $N_{1} \cap N_{2}=\langle 1\rangle$, proving the result. Therefore we assume now that $G$ has a unique minimal normal subgroup $N$. By the induction hypothesis $G / N$ is solvable. Let $p$ be the largest prime dividing the order of $G$. Let $\Phi_{p}(G)$ denote the intersection of all maximal subgroups $M$ of $G$ for which $[G: M]_{p}=1$, (the subgroup $\Phi_{p}(G)$ was introduced by Deskins in [5]). If $N$ is contained in every maximal subgroup $L$ with $[G: L]_{p}=$ 1 , then $N \subseteq \Phi_{p}(G)$ and so $N$ is solvable since $\Phi_{p}(G)$ is solvable ([10, Theorem 7(i)]). Since $G / N$ is solvable this implies that $G$ is solvable, proving the result. So we now suppose that there is some maximal subgroup $L$ of $G$ such that $[G: L]_{p}=1$ and $N$ is not contained in $L$. Since $N$ is the unique minimal normal subgroup of $G$ and $N$ is not contained in $L$, it follows that the core of $L$ is $\langle 1\rangle$. Further, we have that $G=L N$ and $\eta(G: L)=o(N)$. Now, we claim that $[G: L]$ is composite. For, if not, suppose that $[G: L]=r$, a prime. Clearly, $r<p$. Then by considering the permutation representation of $G$ on the $r$ cosets of $L$ and using the fact that the core of $L$ is $\langle 1\rangle$, we obtain that the order of $G$ divides $r$ ! which is absurd since $p$ divides the order of $G$ and $r<p$. Hence [ $G: L]$ is composite. So by the hypothesis we get that $[G: L]=\eta(G: L)$ and consequently

$$
[G: L]=\eta(G: L)=o(N) .
$$


Since $[G: L]_{p}=1$, it follows that $p$ does not divide the order of $N$. Now, let $M$ be any maximal subgroup of $G$ with core $\langle 1\rangle$. Then $G=M N$. Suppose, if possible, that $[G: M]$ is a prime $t$, say. Then clearly $t<p$. By representing $G$ on the $t$ cosets of $M$ and using the fact that the core of $M$ is $\langle 1\rangle$, we obtain that the order of $G$ divides $t$ !, a contradiction as before. Therefore $[G: M]$ is composite. So, $[G: M]=\eta(G: M)$ which in turn is equal to $o(N)$. Thus $G$ has a unique maximal normal subgroup and there is a common divisor of the indices of all the maximal subgroups with core $\langle 1\rangle$. Therefore by using Baer [1, Lemma 3], we get that $G$ has a solvable, normal subgroup $K, K \neq\langle 1\rangle$. Clearly $N \subseteq K$ and so $N$ is solvable. Since $G / N$ is solvable, we now get that $G$ is solvable.

The converse is a direct consequence of Deskins [5, 2.5].

3. Supersolvable groups. First we prove a lemma

LEMMA 3.1. If $M$ is a maximal subgroup of a group $G$ such that $[G: M]$ is a square-free integer then $\eta(G: M)=[G: M]$.

Proof. We use induction on the order of $G$. If $G$ is simple then $\eta(G: M)=o(G)$ and so $o(G)$ is square-free, $G$ is supersolvable and simple, hence $M=1$ and $\eta(G: M)=[G: M]=p$. Assume that $G$ is not simple. Let $N$ be a minimal normal subgroup of $G, N \neq\langle 1\rangle$. If $N \subseteq M$ then by 2.2 we have $\eta(G / N: M / N)=\eta(G: M)$ and since $\eta(G: M)$ is square-free, it follows that $\eta(G / N: M / N)=[G / N$ : $M / N]$ implying that $\eta(G: M)=[G: M]$. Now, suppose that $N$ is not contained in $M$. Then $G=M N$ and $\eta(G: M)=o(N)$, and $o(N)$ is a square-free integer. So $N$ is supersolvable. So $N$ is a cyclic group of prime order. [G:M] $=o(N) / o(M \cap N)$ is a divisor of $\eta(G: M)=o(N)$. So $M \cap N=\langle 1\rangle$ and $[G: M]=o(N)=\eta(G: M)$, proving the result.

In Mukherjee [9, Corollary 2] it was established that a group $G$ is supersolvable if and only if $\eta(G: M)$ is square-free for each maximal subgroup $M$ of $G$. We extend this result by showing the following:

THEOREM 3.2. If $G$ is a group such that $\eta(G: M)$ is square-free for every c-maximal subgroup $M$ then $G$ is supersolvable.

Proof. If every maximal subgroup of $G$ is of prime index, then $G$ is supersolvable. Use induction on the order of $G$. Let $N$ be a 
minimal normal subgroup of $G$. If $M / N$ is a $c$-maximal subgroup of $G / N$ then clearly $M$ is a $c$-maximal subgroup of $G$ and so $\eta(G: M)$ is square-free. Thus $\eta(G / N: M / N)$ is square-free. Therefore by the induction hypothesis, $G / N$ is supersolvable. Now, if $N_{1}$ is a minimal normal subgroup of $G, N \neq N_{1}$, then the same arguments as above yield that $G / N_{1}$ is also supersolvable. Consequently, $G / N \cap N_{1} \simeq G$ is supersolvable, proving the result. Hence we may now assume that $N$ is the unique minimal normal subgroup of $G$. Now if $N$ is contained in every maximal subgroup of $G$, then $N$ is contained in the Frattini subgroup $\Phi(G)$. Since $G / N$ is supersolvable and $N \subseteq \Phi(G)$, it follows that $G / \Phi(G)$ is supersolvable which implies that $G$ is supersolvable. So, we assume that there exists some $M<G$ such that $N$ is not contained in $M$. Then $G=M N$ and $\eta(G: M)=o(N)$. If [ $G: M]$ is composite then by the hypothesis we have that $\eta(G: M)$ is squarefree and so $o(N)$ is square-free which implies that $N$ is supersolvable. So, $N$ must be of prime order and $G$ is supersolvable, proving the theorem. Thus we now assume that for any maximal subgroup $M$ not containing $N,[G: M]$ is a prime and that there exists at least one such $M$.

Let $q$ be the largest prime dividing the order of $G$. Let $\Phi_{q}(G)$ denote the intersection of all maximal subgroups $R$ of $G$ such that [ $G: R]_{q}=1$. Now if $N$ is contained in every maximal subgroup $M_{1}$ of $G$ with $\left[G: M_{1}\right]_{q}=1$ then $N \subseteq \Phi_{q}(G)$ and so $N$ is solvable since $\Phi_{q}(G)$ is solvable [10, Theorem 7(i)]. So $N$, being a solvable, minimal normal subgroup, is elementary abelian. Now taking a subgroup $M$ as in the end of the last paragraph, we have $G=M N$ and so $[G: M]=$ $o(N) / o(M \cap N)$. Also $\eta(G: M)=o(N)$ and $[G: M]$ is a divisor of $\eta(G: M)$ (see the beginning of $\S 2$ ). Consequently $o(M \cap N)=1$, $[G: M]=o(N)$, and so $o(N)$ is a prime. Thus $N$ is cyclic and $G$ is supersolvable, proving the result. Therefore we may now assume that $N$ is not contained in some maximal subgroup $M_{1}$ with $\left[G: M_{1}\right]_{q}=1$. So $G=M_{1} N$. If [ $G: M_{1}$ ] is composite then as in the last paragraph $G$ is supersolvable. Thus we assume that $\left[G: M_{1}\right]$ is a prime, say $r$. Clearly $r<q$. Now consider the permutation representation of $G$ on the $r$ cosets of $M_{1}$. If the core of $M_{1}$ is $\langle 1\rangle$ then it follows that $o(G)$ divides $r$ !, which is absurd. Therefore the core of $M_{1}$ is non-trivial and consequently $N \subseteq M_{1}$, a contradiction. Thus this possibility cannot arise. Hence in all cases $G$ is supersolvable.

4. $p$-solvable and $p$-supersolvable groups. It is proved in Beidleman and Spencer [2, Theorem 1] that a group $G$ is $p$-solvable if and only 
if $\eta(G: M)_{p}=[G: M]_{p}$ for every maximal subgroup $M$ of $G$. This result and a theorem of Mukherjee [9, Theorem 7] for $p$-supersolvable groups are both extended in the following.

TheOREM 4.1. Let $p$ be the largest prime factor dividing the order of a group $G$. Then

(i) $G$ is p-solvable if $\eta(G: M)_{p}=[G: M]_{p}$ for each c-maximal $M$.

(ii) $G$ is $p$-supersolvable if $\eta(G: M)_{p}=[G: M]_{p}=1$ or $p$ for each $c$-maximal $M$.

(Note: The converses of (i) and (ii) are easily seen to be true.)

Proof. (i) Let $G$ be a non $p$-solvable group of minimal order among those satisfying the condition $\eta(G: M)_{p}=[G: M]_{p}$ for each $c$ maximal $M$, with $p$ the maximal prime factor of $o(G)$. Clearly, $G$ is non-abelian and has $c$-maximal subgroups. Furthermore $G$ is not simple. For, if $G$ were simple, consider $L<^{\cdot} G$ with $[G: L]_{p}=1$. If $[G: L]$ is composite then $\eta(G: L)_{p}=o(G)_{p}=1$, contrary to the choice of $p$. So $[G: L]=r$, a prime $<p$, which means that $o(G)$ divides $r$ !, an impossibility. So $G$ is not simple. Let $N$ be a minimal normal subgroup of $G$. If $N$ is not unique then choose another minimal normal subgroup $N_{1} \neq N$. If $p$ divides the orders of both $G / N$ and $G / N_{1}$ then by the minimality of $G$ and using 2.2, both $G / N$ and $G / N_{1}$ are $p$-solvable and so $G / N \cap N_{1} \simeq G$ is $p$-solvable and we are done. Now suppose $p$ does not divide the order of, say, $G / N$, without loss of generality. If $N_{p}$ is a Sylow $p$-subgroup of $N$, by the Frattini argument $G=N_{G}\left(N_{p}\right) N$. If $N_{p} \rtimes G$, choose a maximal subgroup $M$ of $G$ which contains $N_{G}\left(N_{p}\right)$. Then $G=M N$ and clearly $[G: M]_{p}=1$. If $[G: M]$ is composite, then by hypothesis $\eta(G: M)_{p}=[G: M]_{p}=1$ and so $o(N)_{p}=1$, an impossibility. Therefore $[G: M]=s$, a prime $<p$. Now if $\operatorname{Core}_{G}(M)=\langle 1\rangle$, then $o(G)$ divides $s$ !, an impossibility. So $\operatorname{Core}_{G}(M) \neq\langle 1\rangle$. Since $o\left(G /\right.$ Core $\left._{G}(M)\right)$ divides $s$ ! a Sylow $p$-subgroup $P$ of $\operatorname{Core}_{G}(M)$ is a Sylow $p$-subgroup of $G$. Now $\operatorname{Core}_{G}(M) \cap N=\langle 1\rangle$ since otherwise $N \subseteq M$ implying $G=M N=M$, a contradiction. Thus $\operatorname{Core}(M)$ and $N$ centralize each other and so $N_{p} P$ is a $p$-subgroup of $G$ whose order is greater than $o(P)$ which is absurd since $P$ is a Sylow $p$-subgroup of $G$. Hence $N_{p} \triangleleft G$ and so $N_{p}=N$. By the Schur-Zassenhaus theorem $G=N T$ where $T$ is a $p$-complement. Now $G / N$ and $N$ are both $p$-solvable and so $G$ is $p$-solvable, proving the result.

So we now assume that $N$ is the unique minimal normal subgroup of $G$. If $N \subseteq M$ for every $M<^{\cdot} G$ with $[G: M]_{p}=1$, then $N$ is 
contained in their intersection $\Phi_{p}(G)$ so $N$ is solvable and we then obtain easily that $G$ is $p$-solvable, proving the result. So suppose that $N$ is not contained in some $M$ where $M<^{\cdot} G$ and $[G: M]_{p}=1$. Then $G=M N$. If $[G: M]$ is composite then proceeding as in the last paragraph we obtain a contradiction if $\operatorname{Core}_{G}(M)=\langle 1\rangle$; and if $\operatorname{Core}_{G}(M) \neq\langle 1\rangle$ then by the uniqueness of $N, N \subseteq M$ implying $G=M N=M$, a contradiction. Thus $[G: M]$ is composite. By using the hypothesis, $\eta(G: M)_{p}=[G: M]_{p}=1$ and so $o(N)_{p}=1$. Thus $N$ is a $p^{\prime}$-group and $G / N$ is $p$-solvable giving that $G$ is $p$-solvable.

(ii) Let $G$ be a non $p$-supersolvable group of minimal order among those satisfying the condition $\eta(G: M)_{p}=[G: M]_{p}=1$ or $p$ for each $c$-maximal $M$. By (i) $N$ is $p$-solvable. Let $N$ be a minimal normal subgroup of $G$. If $N$ is not unique then choose another minimal normal subgroup $N_{1} \neq N$. If $p$ divides the orders of both $G / N$ and $G / N_{1}$, then as in (i) the result follows easily. Now suppose that $p$ does not divide the order of, say, $G / N_{1}$. Since $N_{1}$ is either a $p$-group or a $p^{\prime}$-group, it follows that $N_{1}$ is a Sylow $p$-subgroup of $G$. By SchurZassenhaus theorem $G=N_{1} T$ where $N_{1} \cap T=\langle 1\rangle$. It is not hard to see that $T<G$. Let $o\left(N_{1}\right)=p^{m}$. If $m>1$, then since [ $\left.G: T\right]=o\left(N_{1}\right)$, $[G: T]$ is composite and by using the hypothesis we obtain that

$$
\eta(G: T)_{p}=[G: T]_{p}=o\left(N_{1}\right)_{p}=1 \text { or } p
$$

which contradicts the assumption $m>1$. Thus $m=1, N_{1}$ is cyclic of order $p$ and since $G / N_{1}$ is trivially $p$-supersolvable, the result follows.

The following result is a generalization of Mukherjee [9, Theorem 8]. We omit the proof which is quite similar to the proof of Theorem 4.1.

THEOREM 4.2. Let $p$ be the largest prime dividing the order of a group $G$. Then $G$ is p-nilpotent if and only if the following conditions hold:

(i) $\eta(G: M)_{p}=[G: M]_{p}=1$ or $p$ for all $c$-maximal subgroups $M$ of $G$.

(ii) If $\eta(G: M)=p$ for some maximal subgroup $M$ of $G$ then $M<\cdot G$.

Acknowledgment. We thank the referee for kind suggestions which helped to improve our exposition. 


\section{REFERENCES}

[1] R. Baer, Classes of finite groups and their properties, Illinois J. Math., 1 (1957), 115-187.

[2] J. C. Beidleman and A. E. Spencer, The normal index of maximal subgroups in finite groups, Illinois J. Math., 16 (1972), 95-101.

[3] P. Bhattacharya and N. P. Mukherjee, A family of maximal subgroups containing the Sylow subgroups and some solvability conditions, Archiv Math., 45 (1985), 390-397.

[4] On the intersection of a class of maximal subgroups of a finite group II, J. Pure and Applied Algebra, 42 (1986), 117-124.

[5] W. E. Deskins, On maximal subgroups, Proc. Symp. Pure Math., Amer. Math. Soc., 1 (1959), 100-104.

[6] D. Gorenstein, Finite Groups, New York 1968.

[7] B. Huppert, Normalteiler und maximale Untergruppen endlicher Gruppen, Math. Zeit., 60 (1954), 409-434.

[8] B. Huppert, Endliche Gruppen, I. Berlin 1967.

[9] N. P. Mukherjee, $A$ note on normal index and maximal subgroups in finite groups, Illinois J. Math., 75 (1975), 173-178.

[10] N. P. Mukherjee and P. Bhattacharya, On the intersection of a class of maximal subgroups of a finite group, Canad. J. Math., 39 (1987), 603-611.

[11] L. R. Nyhoff, The influence on a group of the cofactors and subcofactors of its subgroups, Trans. Amer. Math. Soc., 154 (1971), 459-491.

Received October 15, 1986 and in revised form March 24, 1987.

JAWAHARLAL NeHRU UNIVERSITY

NEW DELHI 110 067, INDIA

AND

UNIVERSITY OF NEBRASKA-LINCOLN

LINCOLN, NE 68588-0115 



\section{PACIFIC JOURNAL OF MATHEMATICS \\ EDITORS}

V. S. VARADARAJAN

(Managing Editor)

University of California

Los Angeles, CA 90024

Herbert Clemens

University of Utah

Salt Lake City, UT 84112

R. FINN

Stanford University

Stanford, CA 94305
HERMANN FLASCHKA

University of Arizona

Tucson, AZ 85721

RAMESH A. GANGOLLI

University of Washington Seattle, WA 98195

VAUGHAN F. R. JONES

University of California

Berkeley, CA 94720
ROBION KIRBY

University of California

Berkeley, CA 94720

C. C. MOORE

University of California

Berkeley, CA 94720

HAROLD STARK

University of California, San Diego

La Jolla, CA 92093

\section{ASSOCIATE EDITORS}
R. ARENS
E. F. BECKENBACH
B. H. NEUMANN
F. WOLF
K. YOSHIDA
(1906-1982)

\section{SUPPORTING INSTITUTIONS}

UNIVERSITY OF ARIZONA

UNIVERSITY OF BRITISH COLUMBIA

CALIFORNIA INSTITUTE OF TECHNOLOGY

UNIVERSITY OF CALIFORNIA

MONTANA STATE UNIVERSITY

UNIVERSITY OF NEVADA, RENO

NEW MEXICO STATE UNIVERSITY

OREGON STATE UNIVERSITY
UNIVERSITY OF OREGON UNIVERSITY OF SOUTHERN CALIFORNIA

STANFORD UNIVERSITY

UNIVERSITY OF HAWAII

UNIVERSITY OF TOKYO

UNIVERSITY OF UTAH

WASHINGTON STATE UNIVERSITY

UNIVERSITY OF WASHINGTON 


\section{Pacific Journal of Mathematics \\ Vol. 132, No. $1 \quad$ January, 1988}

Scott W. Brown, Full analytic subspaces for contractions with rich spectrum ...1

Robert Main Burton, Jr. and Tae-Sung Kim, An invariance principle for

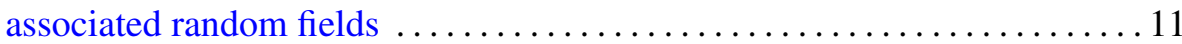

Ana M. Viola-Prioli and Jorge Viola-Prioli, Rings whose kernel functors

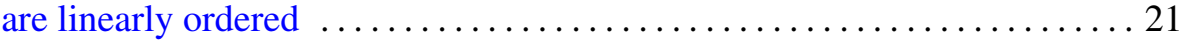

David E. Handelman, Representing polynomials by positive linear

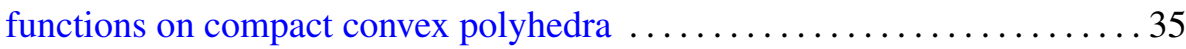

Patrick Keef, On the Tor functor and some classes of abelian groups . .....66

Dennis R. Malm, Simplicity of partial and Schmidt differential operator

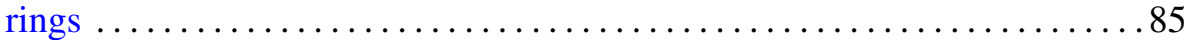

José M. Montesinos and Carmen Safont, On the Birman invariants of

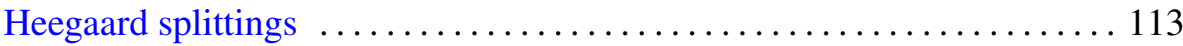

N. P. Mukherjee and Prabir Bhattacharya, The normal index of a finite group

Mario Petrich and Norman R. Reilly, Semigroups generated by certain operators on varieties of completely regular semigroups

Robert Tijdeman and Lian Xiang Wang, Sums of products of powers of given prime numbers

Joel Larry Weiner, First integrals for a direction field on a simply connected plane domain 